\title{
CONSENTIMIENTO INFORMADO EN LAS PRESTACIONES DE SALUD
}

\author{
Gabriel Hernández Paulsen¹, Felipe Chahuán Zedan²
}

Resumen: Por regla general, las prestaciones de salud requieren el consentimiento informado del paciente. Los fundamentos éticos detrás de esta exigencia son la autonomía del paciente para decidir sobre su propio cuerpo y el respeto a su dignidad, que impide instrumentalizarlo. La manera en que el ordenamiento jurídico materializa estos fines es consagrando deberes de información y de consejo, y disciplinando las condiciones bajo las cuales debe prestarse el consentimiento. Jurídicamente, estos deberes delimitan la relación médico-paciente y ayudan a definir hipótesis de responsabilidad civil. En este artículo se revisan las condiciones para la satisfacción de estos deberes, así como los supuestos en que su infracción pueden dar lugar a responsabilidad civil.

Palabras clave: consentimiento informado, dignidad y autonomía del paciente, deberes de información y de consejo, responsabilidad civil

\section{Informed consent in health care services}

Abstract: As a general rule, medical interventions require to be informingly consented by the patient. This requirement is ethically founded in the autonomy of the patient to decide on his own body and the respect for his dignity that prevent to instrumentalise it. The way in which the law materialises those ends is by implementing duties to inform and duties to advise, and regulating the conditions under which the consent must be given. Legally, those duties delimit the relation physicianpatient and give guidance to define hypothesis of civil liability. In this article, we revise the conditions for the satisfaction of those duties and the cases in which their breach give rise to civil liability.

Keywords: Informed consent, dignity and autonomy of the patient, duty to inform and duty to advise, civil liability

\section{Consentimento informado nas prestaçóes de saúde}

Resumo: Por regra general, as prestações de saúde requerem o consentimento informado do paciente. Os fundamentos éticos por trás dessa exigência sáo a autonomia do paciente para decidir sobre seu próprio corpo e o respeito à sua dignidade, que impede instrumentalizá-lo. A maneira em que o ordenamento jurídico materializa estes fins é consagrando deveres de informação e de aconselhamento, e disciplinando as condiçóes sob as quais deve prestar-se o consentimento. Juridicamente, estes deveres delimitam a relação médico-paciente e ajudam a definir hipóteses de responsabilidade civil. Neste artigo se revisam as condiçóes para a satisfaçáo destes deveres, assim como os pressupostos em que sua infraçáo podem dar lugar à responsabilidade civil.

Palavras chave: consentimento informado, dignidade e autonomia do paciente, deveres de informação e de aconselhamento, responsabilidade civil

\footnotetext{
${ }^{1}$ Departamento de Derecho Privado, Facultad de Derecho, Universidad de Chile. ORCID ID 0000-0002-3180-8067.

Este artículo está vinculado con el Proyecto Fondecyt de Iniciación 11180826, del que Gabriel Hernández es Investigador Responsable. Correspondencia: ghernan@derecho.uchile.cl

${ }^{2}$ Departamento de Derecho Privado, Facultad de Derecho, Universidad de Chile. ORCID ID 0000-0003-3265-9056.

Correspondencia: Ichahuan@derecho.uchile.cl
} 


\section{Introducción}

Un precedente fundacional en materia de consentimiento informado es el caso Schloendorff. El juicio fue iniciado por un paciente que aceptó someterse a un examen bajo anestesia para determinar si un tumor era benigno o maligno. El médico concluyó que el tumor era cancerígeno y decidió extirparlo, provocando una gangrena y la amputación de algunos dedos de la mano. El afectado alegó que la extirpación se hizo sin su consentimiento, ante lo que la Corte de Apelaciones de Nueva York resolvió que "toda persona adulta y con una mente sana tiene derecho a determinar qué puede ser hecho con su cuerpo" y, en consecuencia, "el médico que realiza una operación sin su consentimiento comete un asalto por el que resulta responsable de los daños causados"3.

Las consideraciones éticas subyacentes a dicha decisión son la dignidad de los pacientes y su autonomía para gobernar su vida y cuerpo, cuyo reconocimiento ha significado un replanteamiento de su relación con el médico y una regulación que impone a los prestadores de salud numerosos deberes, entre los que destacan los de información y de consejo. El propósito de imponerles tales deberes es que el paciente manifieste su voluntad con conocimiento acerca de las prestaciones de salud, sobre todo de sus riesgos. Aquella voluntad se denomina "consentimiento informado" y está regulada por la ley No 20.584 , de derechos y deberes de los pacientes (LDDP), que establece sus requisitos de configuración.

Aparte de propender a dar vigencia a los señalados fundamentos, las reglas sobre consentimiento informado configuran buena parte del contenido de la relación médico-paciente $y$, en consecuencia, determinan los casos y la extensión en que el prestador puede comprometer su responsabilidad civil.

En lo que sigue, revisaremos y sistematizaremos, bajo consideraciones éticas y jurídicas, los fundamentos y requisitos del consentimiento informado en el ámbito de las prestaciones de salud, así como los supuestos de responsabilidad civil que puedan presentarse por infracción de las reglas

${ }^{3}$ Sentencia Schloendorff $v$ Society of New York Hospital, 105 NE 92 (NY 1914) [traducción libre]. que lo disciplinan.

\section{Fundamentos y fines}

El consentimiento informado es una concreción de libertades constitucionales ${ }^{4}$, en especial aquellas que reconocen y protegen un estatus de dignidad $(1,2)$ y una esfera de autonomía a las personas(3:57)5. Típicamente, se ha destacado el nexo con la libertad de conciencia, habiéndose fallado en Chile, por ejemplo, que una transfusión de sangre efectuada sin el consentimiento del paciente puede significar una vulneración de dicha libertad ${ }^{6}$.

La manera en que el ordenamiento jurídico materializa estos fundamentos éticos y constitucionales es configurando deberes de información y de consejo que, a su vez, son manifestaciones de los principios jurídicos de autonomía privada, buena fe y corrección de asimetrías (5:2475).

Tales principios justifican que el paciente tenga el derecho a recibir información que, si no se le puede proporcionar directamente - por ejemplo, en caso de problemas emocionales o de entendimiento, alteración de conciencia o urgencia-, la deberá recibir su representante o cuidador familiar, allegado o persona de confianza designada- (DS 38, artículo 4). Aunque el principio de autonomía supone que, por regla general, es el paciente quien debe decidir, no implica que no pueda ser guiado por un experto, siendo esperable que, atendida la profesionalidad del prestador, pueda orientarlo para que tome la mejor determinación posible, de acuerdo con su plan de vida y estado de salud. Por esto, la relación entre ambos se ha caracterizado como un diálogo dirigido a asegurar que el paciente comprenda su condición de salud para decidir, lo que supone que el médico informe, pero también aconseje al paciente de manera personalizada ${ }^{7}$.

\footnotetext{
${ }^{4}$ En Espańa, sentencia del Tribunal Supremo (Sala de lo Civil), 12.1.2001, No 3688-1995; en Francia, Cass. $1^{\text {er }}$ civ., 9.10.2001; en Chile, Corte Suprema, 28.1.2011, rol No 5849-2009.

${ }^{5}$ Se han presentado dudas respecto de si los deberes relativos al consentimiento informado permiten una mayor autonomía debido a que los pacientes, en general, no tendrían la aptitud para procesar la información recibida(4).

${ }^{6}$ Corte Suprema, 13.12.2019, rol No 24199-2019.

${ }^{7}$ En Reino Unido, sentencia Montgomery v Lanarkshire Health Board [2015] UKSC 11 [para 90]; en Chile, Corte de Apelaciones de
} 
Con todo, la protección de la dignidad y autonomía no son las únicas consideraciones que rigen la relación médico-paciente. En casos específicos, la ley permite a los prestadores de salud actuar pese a que el paciente o su representante haya manifestado una voluntad contraria. Son supuestos en que la autoridad del médico "triunfa" frente a la libertad del paciente. Así, el derecho a prestar un consentimiento informado, en ocasiones, es subordinado a otros fines. Se trata de casos en que el interés en proteger la autonomía cede ante razones éticas y jurídicas consideradas de mayor peso. Por ejemplo, en el caso de procedimientos o tratamientos cuya falta se traduce en la aceleración artificial de la muerte, la realización de prácticas eutanásicas o el auxilio al suicidio (LDDP, artículos 14 y 16); y en el del médico que puede ignorar la negativa del paciente a someterse a un procedimiento si, previa consulta al comité ético, concluye que su decisión lo expone a dańos graves o compromete la salud pública (LDDP, artículos 15 a) y 16). Atendido que se trata de reglas que limitan el principio de autodeterminación —que inspira la LDDP-, no debiesen extenderse a hipótesis distintas de las señaladas(6:735), como en ocasiones han hecho los tribunales, al postergar el derecho a prestar un consentimiento informado frente a otros derechos constitucionales estimados de mayor rango, e imponer, incluso, restricciones superiores a las legales ${ }^{8}$.

También en supuestos en que alguna incapacidad impide que el paciente pueda decidir o que se consulte a su representante, la LDDP autoriza a los prestadores a actuar prescindiendo de su voluntad (artículo 15). A diferencia de los casos anteriores, esta autorización no posterga el principio de la autonomía al ser imposible que el afectado la ejerza, basándose la ley en el principio del reguardo de la vida o salud del paciente, que guía el comportamiento médico?.

Además de aspirar a dar vigencia a los mencionados principios, las reglas sobre consentimiento informado tienen por fin delimitar el objeto de la relación con el paciente, posibilitando que co-

Santiago, 18.6.2015, rol No 5396-2014; en Francia(5:2475).

${ }^{8}$ Corte Suprema 20.4.2015, rol No 5.027-2015.

${ }^{9}$ Bajo una perspectiva distinta, se ha argumentando que se trata de un criterio que refuerza la autonomía del paciente, apelando a la idea de consentimiento hipotético(6:734). nozca sus derechos y que el prestador sepa cuáles son sus obligaciones y los riesgos aceptados por aquel, así como las eventuales modificaciones de procedimiento que asume ${ }^{10}$. Como consecuencia, dichas reglas contribuyen a determinar los casos en que el prestador resultará civilmente responsable, y su extensión.

\section{Requisitos de los deberes asociados al con- sentimiento informado}

El cumplimiento del deber de informar supone que los antecedentes entregados al paciente satisfagan determinadas cualidades, que le permitan conocer el procedimiento a que se someterá. En este sentido, por ejemplo, la Corte de Casación francesa ha señalado que la información debe ser fidedigna, ilustrativa y apropiada ${ }^{11}$. En Chile, la LDDP exige que la información sea suficiente, oportuna, adecuada, comprensible y veraz.

Que la información sea suficiente significa que debe contener todos los elementos que permitan al paciente tomar una decisión informada. Que sea oportuna implica que, en la medida de lo posible, debe proporcionarse con una antelación razonable a la prestación. Que sea adecuada supone su adaptación a las circunstancias y capacidades de quien la recibe. En la medida de que la economía del comportamiento y la psicología conductual coinciden en que la capacidad para procesar información es limitada(7), especialmente cuando existe una carga emocional, debe tenerse en cuenta que no siempre más información permite cumplir con el objetivo de mejorar la posición del paciente ${ }^{12}$. Por esto, la información debe entregarse de manera personalizada y ser comprensible, es decir, entendible para el paciente, lo que es consistente con la afirmación de que, aparte de recaer sobre los prestadores el deber de informarle, cargan con el de aconsejarle. Por último, que la información sea veraz supone que debe ser cierta y no inducir a engaño o error.

La LDDP no es consistente al determinar en qué casos deben cumplirse las aludidas calidades. Tra-

\footnotetext{
${ }^{10}$ En Francia, Cass. $1^{\text {er }}$ civ., 14.10.1997; en Chile, Corte Suprema, 28.1.2011, rol No 5849-2009.

${ }^{11}$ Cass. 1er civ., 14.10.1997; Cass. 1er civ., 9.10.2001.

${ }^{12}$ Un reconocimiento de esta limitación en materia médica en sentencia Montgomery v Lanarkshire Health Board [2015] UKSC 11.
} 
tándose de los términos y condiciones de la prestación de salud, el artículo 8 exige que la información sea proporcionada al paciente de manera suficiente, oportuna, veraz y comprensible. El deber es impuesto a los prestadores institucionales e individuales, que son los establecimientos y personas que, de modo dependiente o independiente, colaboran directa o indirectamente en la prestación de salud (artículo 3). En cambio, respecto de la información sobre el estado de salud del paciente y su diagnóstico, solo se exige al médico $u$ otro profesional tratante que la entregue en forma oportuna y comprensible (artículo 10). El artículo 14 agrega que la información debe ser adecuada, pero no dice nada sobre su veracidad ni suficiencia. En todo caso, pese a la falta de mención de la veracidad, es lógico que no pueda considerarse informado el consentimiento si ha sido prestado sobre la base de antecedentes erróneos ${ }^{13}$, principalmente porque su fundamento es la dignidad y la autonomía del paciente. Por su parte, respecto de la suficiencia, en ciertos casos podría estimarse que la entrega de información incompleta resulta 'inocua'14, en relación con lo cual nuevamente debe tenerse en cuenta la necesidad de satisfacer los requerimientos de los principios de dignidad y autonomía.

El contenido de la información que tiene derecho a recibir el paciente depende de su edad, condición personal y emocional (LDDP, artículo 10, inciso $1^{\circ}$ ), debiendo, por tanto, ser personalizada. Se trata de una exigencia que, aun cuando debe ser satisfecha por el prestador, supone la cooperación del paciente, sus familiares o representantes legales, quienes tienen la carga de proporcionar información veraz sobre determinados aspectos ("necesidades y problemas de salud" y "todos los antecedentes que conozcan o les sean solicitados para su adecuado diagnóstico y tratamiento" LDDP artículo 36- $)^{15}$. Pese a que la LDDP no lo establece, los prestadores tienen el deber de recabar del paciente los antecedentes pertinentes

\footnotetext{
${ }^{13}$ Corte Suprema, 1.6.2017, rol No 87914-2016.

${ }^{14}$ Corte Suprema, 2.10.2013, rol No 4325-2013.

${ }^{15}$ Se ha dicho también que se trata de un deber del paciente( $\left.8: 47\right)$. Nos parece que la calificación correcta es estimar que se trata de una carga. Estas se diferencian de un deber jurídico porque no tienen por objeto la conducta ajena, sino la del titular del derecho. La consecuencia de su infracción, por lo tanto, es usualmente la pérdida del derecho o la imposibilidad de ejercerlo.
}

para informarlo adecuadamente, conclusión que se sustenta sobre todo en el principio de la dignidad y en la concepción dialogal de la relación médico-paciente.

La LDDP detalla el tipo de información que debe entregarse al paciente, distinguiendo según el agente obligado a informar y el momento en que debe hacerlo. Antes de un procedimiento o intervención médica, los prestadores institucionales deben informar al paciente acerca de distintos aspectos relativos a las condiciones y términos de la prestación (como los valores y las condiciones previsionales, entre otros). Asimismo, el médico o profesional tratante debe informar al paciente sobre su estado de salud, diagnóstico, alternativas de tratamiento, riesgos, pronóstico y proceso previsible del postoperatorio (LDDP, artículos 8 y 10). Al finalizar una hospitalización, el médico debe informar al paciente sobre la producción de todo 'evento adverso' (LDDP, artículo 4), la identificación del tratante principal, el periodo de tratamiento, el diagnóstico de ingreso y alta, los resultados relevantes de exámenes y procedimientos, la medicación administrada y que se debe consumir, y los cobros (LDDP, artículo 11) ${ }^{16}$.

Como es lógico, un punto crítico en estos deberes de información son los riesgos asociados al procedimiento o prestación. Sin embargo, es difícil determinar qué se considerará una información y consejo adecuado en relación con ellos. La Corte Suprema ha señalado que el deber de informar incluye los riesgos personales y probables según el estado de avance de la ciencia y la experiencia del médico tratante ${ }^{17}$, o los riesgos usuales o esperados ${ }^{18}$. Por otro lado, un análisis general a la luz de algunos ordenamientos extranjeros muestra que las respuestas pueden variar respecto de la técnica o cláusulas usadas para determinar cuáles riesgos deben informarse y cuáles no. En Francia, por ejemplo, la Corte de Casación ha estimado que deben informarse los riesgos frecuentes y todos los graves, aunque sean excepcionales(9:51),

\footnotetext{
${ }^{16}$ Aunque la ley impone la obligación de informar al paciente acerca de tratamientos alternativos, la Corte Suprema, en un fallo dividido, sentenció que, pese a ello, los médicos no tienen el deber de informar a su respecto si han aplicado el tratamiento recomendado a la luz de la lex artis. Corte Suprema, 6.7.2018, rol No 8990-2018.

${ }^{17}$ Corte Suprema, 18.6.2015, rol No 5396-2014.

${ }^{18}$ Corte Suprema, 28.1.2011, rol No 5849-2009.
} 
quedando excluidos aquellos riesgos graves no conocidos debido al estado de desarrollo de la ciencia $^{19}$. No obstante, el Código de Salud Pública de dicho país relajó tal criterio, ordenando informar los riesgos frecuentes y los serios normalmente predecibles (artículo 1111-2). En Reino Unido, en cambio, si bien tradicionalmente la Corte Suprema buscó determinar cuál es la información que un consejero médico responsable comunicaría ${ }^{20}$, hoy invoca el estándar de materialidad de la información, referido a aquellos riesgos que una persona razonable, en la posición del paciente, probablemente le asignaría importancia, y a aquellos que el médico sabe o debería saber que el paciente le atribuiría importancia ${ }^{21}$.

En cuanto a la forma de entrega de la información, la real consideración de la autonomía del paciente requiere que el medio usado para transmitirla sea idóneo a las circunstancias del destinatario. Tratándose de los prestadores institucionales de salud, el artículo 8 de la LDDP señala que dicho medio puede ser visual, verbal o escrito, estableciendo formas específicas de transmisión de ciertos tipos de información. Por ejemplo, la de poner y mantener en un lugar público y visible una carta de derechos y deberes de las personas en relación con la atención de salud; y la de que la información sobre aranceles y procedimientos de cobro sea escrita, con detalle de los insumos, medicamentos, exámenes, derechos de pabellón, días de cama y honorarios de quienes realizaron la atención (artículo 11). En el caso de intervenciones quirúrgicas, procedimientos diagnósticos y terapéuticos invasivos y, en general, para la aplicación de procedimientos que conlleven un riesgo relevante y conocido para la salud del afectado, la información deberá constar por escrito en la ficha clínica (artículo 14).

La carga de probar que la información necesaria para que el paciente manifieste su consentimiento ha sido entregada es de los prestadores, pero la LDDP la facilita al establecer la presunción de que ha sido obtenido si "hay constancia de su firma en el documento explicativo del proce-

\footnotetext{
${ }^{19}$ Cass. $1^{\text {er }}$ civ., 2.10.2002.

${ }^{20}$ Sentencia Bolam v Friern Hospital Management Committee [1957] 1 WLR 583.

${ }^{21}$ Sentencia Montgomery $v$ Lanarkshire Health Board [2015] UKSC 11.
}

dimiento o tratamiento al cual deba someterse" (artículo 8). La presunción puede ser desvirtuada por el paciente si acredita que, pese a dicha firma, no ha recibido la información de la manera que exige la ley.

Solo una vez que el paciente recibe la información surge la posibilidad de que preste su consentimiento, ya que solo así puede entenderse que este ha sido ejercido en forma verdaderamente libre, voluntaria, expresa y con conocimiento de causa. Aunque el objeto de la LDDP es precisamente obtener este consentimiento autónomo, en ella se incluyen algunas disposiciones que atienden a la posición ética y jurídica del médico en relación con la decisión del paciente. En primer lugar, se autoriza al médico a pedir la opinión del comité de ética si duda acerca de la competencia del paciente que manifiesta su voluntad, o estima que su decisión le expone a graves daños a su salud o, incluso, a la muerte. En segundo lugar, le permite no continuar como encargado del tratamiento si discrepa de la decisión del paciente, siempre que asegure que su responsabilidad será asumida por otro profesional calificado, en atención al caso específico (artículo 17).

Por regla general, el consentimiento puede prestarse en forma verbal, pero deberá constar por escrito en caso de intervenciones quirúrgicas, procedimientos diagnósticos y terapéuticos invasivos, y de aplicación de procedimientos que conlleven un riesgo relevante y conocido para la salud. En estos casos, el otorgamiento de información y la aceptación o el rechazo del paciente deberán constar por escrito en su ficha clínica (LDDP, artículo 10). No obstante, la Corte Suprema ha fallado que no es necesario que el consentimiento escrito del paciente se encuentre en su ficha clínica si consta en otros instrumentos ${ }^{22}$.

Naturalmente y sobre la base de los principios de dignidad y autonomía, si el paciente hubiera expresado su voluntad de no ser tratado, quisiera interrumpir el tratamiento o se negara a cumplir las prescripciones médicas, el establecimiento, a propuesta del profesional tratante y previa consulta al comité de ética, está facultado para decretar el alta forzosa (LDDP, artículo 18).

\footnotetext{
${ }^{22}$ Corte Suprema 13.6.2018, rol No 37438-2017.
} 


\section{Deberes asociados al consentimiento infor- mado y responsabilidad civil}

El incumplimiento de las reglas relativas al consentimiento informado puede desencadenar responsabilidad civil, siendo relevante, por tanto, determinar cuándo han de entenderse satisfechos los deberes asociados a él y cuándo no $^{23}$.

Según vimos, los antecedentes que tienen que entregar los prestadores de salud dependen del caso concreto. Definida la información que ha de proporcionarse al paciente, debe necesariamente entregársele, correspondiendo este deber, por tanto, a una obligación de resultado $(9: 51)^{24}$, sin perjuicio de que la evaluación relativa a si se cumplieron los requisitos exigidos a la información (características, contenido y medio de traspaso) constituya un juicio normativo sujeto a cierta flexibilidad. Tratándose del deber de consejo, la evaluación de si se cumplió constituye un juicio que exige demostrar que se hicieron esfuerzos razonables para que el paciente comprendiera la recomendación.

El prestador que realiza un tratamiento o intervención sin obtener el consentimiento informado del paciente $-o$ de sus representantes, cuando corresponda-, incumple su deber, pudiendo dar lugar a responsabilidad civil ${ }^{25}$. De manera que dicho consentimiento opera como una autorización para realizar un procedimiento, que, en su ausencia, no podría efectuarse(10). Así, a diferencia del rol del consentimiento en el ámbito contractual general, cuyo valor radica en su poder para generar obligaciones, en materia médica opera, adicionalmente, como una autorización que desplaza el deber de abstención del médico, volviendo permisible lo que de otro modo no lo sería(11). Solo constituyen excepción aquellos casos en que la ley autoriza al médico a no obtener el consentimiento o a actuar contra la voluntad del paciente.

${ }_{23}$ Además de tratarse de deberes legalmente regulados, se ha considerado que su cumplimiento es uno de los requerimientos de la lex artis. En Chile, Corte Suprema 27.6.2013, rol No 2332-2012; en España, sentencia del Tribunal Supremo (Sala ContenciosoAdministrativa) 20.9.2005, № 5078/2002.

${ }^{24}$ También se ha señalado que el deber de información corresponde a una obligación de medios(10).

25 Ahora bien, el cumplimiento de los deberes asociados al consentimiento informado igualmente puede dar lugar a la responsabilidad del prestador por negligencia desencadenante de un daño, si el procedimiento informado se realiza infringiendo algún deber de la lex artis, Corte Suprema, 28.1 2011, rol No 5849-2009.
En principio, el médico será civilmente responsable cuando su intervención se traduce en la materialización de un riesgo no informado debidamente o producido como efecto de un procedimiento no consentido por el paciente, aun cuando el perjuicio no se haya causado por una prestación de salud deficiente. Por el contrario, el prestador puede resultar exonerado si el detrimento provocado ha sido fruto de un riesgo informado, en la medida de que se han observado los cuidados debidos ${ }^{26}$.

Pero, aunque en los supuestos en que el médico no observa los deberes asociados al consentimiento informado hay una negligencia, afirmar la responsabilidad civil del médico plantea difíciles preguntas en materia de daño y de causalidad. En efecto, surgen dudas relativas, primero, a la identificación del daño y, segundo, en relación con el vínculo de causalidad entre el daño sufrido y la infracción al deber de información y consejo del médico.

\section{Identificación del daño}

La atribución de responsabilidad puede enfrentar problemas en la identificación del daño en supuestos de incumplimiento de los deberes asociados al consentimiento informado. En su caso, si el procedimiento es el recomendado por la lex artis y se desarrolla con la diligencia debida, no es tan clara la conclusión de que el empeoramiento del estado de salud sea un daño indemnizable ${ }^{27}$. En efecto, en muchos casos, la decisión informada de no intervenir puede ser más perjudicial para la salud. Por ejemplo, cuando se trata de la extirpación de un tumor cerebral que se realizaría bajo los estándares de la lex artis con el riesgo de provocar disminución de facultades motoras, en que la falta de intervención podría generar que el tumor se expanda rápidamente y acelere la muerte.

No obstante, el argumento de que el eventual estado de salud en que quedaría el paciente sería peor que aquel en que quedaría en virtud de una intervención no consentida, no debiese ser acogido para descartar el daño. En estos casos hay un daño, porque objetivamente un interés legítimo

\footnotetext{
${ }^{26}$ Corte Suprema, 14.1.2015, rol No 16318-2013.

${ }^{27}$ Corte Suprema, 2.10.2013, rol No 4325-2013 (afirmando que la infracción a un deber de información por sí misma es inocua).
} 
del paciente ha resultado lesionado (en nuestro ejemplo, el interés de no ver afectadas las facultades motoras). La circunstancia de existir un peligro más intenso, que no se concretó gracias a la intervención, no elimina el hecho de que en la salud del paciente se haya materializado otro riesgo, aunque comparativamente pueda estimarse de menor entidad que otro ${ }^{28}$. Por lo tanto, el deterioro en la salud del paciente, aun cuando no se deba a una prestación defectuosa, debería ser indemnizado por el prestador ${ }^{29}$, bajo el entendido de que, por no informar al paciente, asumió el riesgo del daño(12).

Más difíciles son aquellos casos en que la intervención no consentida resulta plenamente exitosa, sin que implique un deterioro en la salud del paciente. Una alternativa es sostener que aun en estas circunstancias habría daño, consistente en la supresión de la oportunidad de decidir exponerse a un riesgo. Tanto en los casos en que el daño se materializa como en los que no, el paciente ha sido privado de su oportunidad de decidir. De este modo, la realización de una intervención o tratamiento no consentido de manera informada sería un detrimento a la dignidad y esfera de autonomía de la persona(13). Así lo ha reconocido, por ejemplo, la Corte de Casación france$\mathrm{sa}(5: 2477)$.

Hay razones para tomar con cuidado la alternativa anterior. Dar lugar a la responsabilidad de los prestadores por incumplimiento de los deberes asociados al consentimiento informado en supuestos en que no hay un efecto adverso en la salud, podría producir un impacto en la estructura de la responsabilidad civil. En efecto, considerar que el daño a la autonomía es indemnizable, pese a que no haya un agravamiento subsecuente del paciente, supone asimilar la negligencia del médico con el daño. Dicho de otro modo, si el daño está constituido por la lesión negligente al derecho a prestar un consentimiento informado, y no por una consecuencia moral o material distinta, la negligencia y el daño se confundirían, al estar

\footnotetext{
${ }^{28}$ Así, se ha sosteniendo que el dańo se identifica comparando una situación anterior con otra posterior, y no comparando una situación real con una situación hipotética(12).

${ }^{29}$ Este es el criterio seguido en España. Véase la sentencia del Tribunal Supremo (Sala de lo Civil), 26.9.2006, res. No 927. Véase también Corte Suprema 27.6.2013, rol No 2332-2012.
}

representada la primera por el incumplimiento de los deberes asociados al consentimiento informado y, el segundo, por la misma lesión al señalado derecho provocada por tal incumplimiento. Esta confusión de ambas categorías ha llevado a algunos a reconocer un daño indemnizable solo en la medida de que un riesgo a la salud se haya materializado; de otro modo, se dice, solo habría una afectación irrelevante a la autodeterminación(9:59).

En verdad, no parece adecuado señalar que, de no materializarse ningún riesgo tras la intervención no consentida, la afectación a la esfera de autonomía del paciente es irrelevante. Su relevancia consiste en haberse vulnerado un ámbito de decisión del paciente, que es precisamente el fundamento ético del consentimiento informado. Con todo, la relevancia jurídica de tal infracción no necesariamente debería traducirse en responsabilidad civil ${ }^{30}$, cuya aplicación supone la existencia de un daño consecuencial al ilícito. Quizás una manera menos intrincada de enfrentar el problema sería encontrar el daño no directamente en la afectación a la autonomía del paciente, sino en un perjuicio moral distinto representado por el detrimento psicológico que podría sufrir quien ve intervenido su cuerpo sin su consentimiento ${ }^{31}$, en la medida en que este daño sea acreditado.

\section{Causalidad}

Además de la materialización de un daño, para que haya lugar a la responsabilidad debe acreditarse la causalidad $^{32}$, que también genera dudas relevantes en supuestos de incumplimiento de los deberes asociados al consentimiento informado. Ante todo, es posible que, de haber el médico entregado debidamente la información al paciente, este hubiera consentido la intervención, por lo que igualmente se habría realizado. En este tipo

\footnotetext{
${ }^{30}$ Explorando la responsabilidad penal del médico por infracción al consentimiento informado en casos en que no se materializa un riesgo para el paciente(14).

${ }^{31}$ En Francia, Cass. $1^{\text {er }}$ civ., 3.6.2010. Jurisprudencia anterior, con todo, había negado la plausibilidad de un daño psicológico, Cass. $1^{\text {er }}$ civ., 6.12.2007.

${ }^{32}$ Corte de Apelaciones de Santiago, 18.6.2015, rol No 5396-2014 (descartando el vínculo de causalidad pese a reconocer la existencia del dańo y la infracción al deber de información). Véase también Corte de Apelaciones de Concepción, 30.5.2013, rol No 1473 2012.
} 
de supuestos se ha planteado que operaría un consentimiento hipotético, que podría eximir de responsabilidad al médico(6:740).

Avalar un consentimiento hipotético, sin embargo, sería objetable a la luz de los fundamentos y fines del consentimiento informado, ya que la necesidad de obtenerlo busca afirmar la dignidad y autonomía del paciente, que son socavadas si se justifica que la suposición acerca de lo que habría determinado reemplaza su decisión efectiva(9:56-69).

Pese a que dicha objeción es plausible desde el punto de vista de la culpabilidad, no necesariamente lo es desde la causalidad. En otras palabras, la noción de consentimiento hipotético puede tener valor si se predica respecto de la causalidad, mas no de la culpabilidad, ya que la suposición de la voluntad del paciente no borra la falta del médico, pero permite plantear dudas respecto del escenario contrafactual, que es la manera generalizada de construir juicios sobre causalidad. De este modo, afirmar la causalidad en caso de incumplimiento de los deberes asociados al consentimiento informado requiere sostener que, de haberse cumplido dichos deberes, el daño no se hubiera producido. Básicamente, esto supone afirmar que el paciente, de haber sido informado adecuadamente, no habría prestado su consentimiento, evaluación que debe estar referida al momento en que debió otorgar su voluntad y no al de materialización de los riesgos.

Pero en algunas ocasiones puede resultar extremadamente complicado afirmar con certeza que la falta de dicho consentimiento fue la causa, en sentido fáctico y jurídico, de la supresión de la posibilidad de evitar el daño final. Una manera de afrontar la dificultad es asumir que ha habido una pérdida de oportunidad para el paciente de evitar la materialización del riesgo ${ }^{33}$, siendo el monto de la indemnización solo proporcional al daño final sufrido(15:104). En efecto, bajo la noción de pérdida de oportunidad el sistema de responsabilidad civil ha abordado los supuestos en que, debido a limitaciones epistemológicas, resulta imposible alcanzar certeza sobre la causa real del daño y, en particular, saber si podría haberse evitado de no mediar la conducta negligente(16,6:399).

\footnotetext{
33 En Chile, Corte Suprema, 26.1.2012, rol No 8352-09. En Francia, Cass. 1er civ., 13.2.2007.
}

\section{Conclusión}

El consentimiento informado del paciente, por regla general, demarca el ámbito dentro del cual una intervención o procedimiento médico pueden considerarse jurídicamente justificados. Su fundamento ético consiste en que el paciente es quien decide sobre su propio cuerpo (esto es, quien tiene autonomía) y, en consecuencia, el médico está impedido de instrumentalizarlo para alcanzar otros fines (esto es, debe respetar su estatus de dignidad). Solo en ocasiones excepcionales la legislación permite al médico actuar a pesar de una decisión contraria del paciente, en consideración a razones que derrotan en circunstancias específicas a la autonomía. Más allá de los supuestos específicamente legislados, la decisión del médico siempre debiera estar delimitada por la voluntad informada del paciente.

Las decisiones de salud suelen estar mediadas por la asimetría en la relación médico-paciente y por la carga emocional que usualmente suponen. En este contexto, el consentimiento informado solo puede lograrse mediante el establecimiento de deberes de información y consejo. En el ámbito de la salud, la legislación regula con detalle el tipo de información que los prestadores deben entregar y las condiciones para que el consentimiento del paciente sea eficaz.

Por una parte, la complejidad de tal tipo de decisiones exige pautas de comportamiento que sean claras y precisas. Pero, por otra, también es necesario algún margen de flexibilidad para que el médico adapte la información según las circunstancias personales del paciente. La manera de lograr este equilibrio es regulando el tipo de información que como mínimo debe entregarse (obligación de resultado), pero exigiendo, a la vez, que se entregue con determinadas cualidades. En el caso de la legislación chilena, la información debe ser suficiente, oportuna, adecuada, comprensible y veraz. Atendidas las limitaciones humanas para procesar una gran cantidad de antecedentes, en muchos casos un exceso de información puede afectar la capacidad del paciente para comprenderla. En estas circunstancias, es esperable que los médicos también den un consejo que permita una decisión efectivamente libre e informada.

En la medida en que el consentimiento informado 
traza las fronteras entre una intervención médica lícita e ilícita, su infracción puede dar lugar a la responsabilidad civil del médico. Aquí se ha sostenido que la extensión de tal responsabilidad no siempre será igual, planteándose algunas preguntas difíciles respecto de la identificación del daño y del vínculo causal. Ante todo, la responsabilidad debiera cubrir los efectos adversos que puedan ser causalmente vinculados con una intervención no consentida, aun cuando se trate de riesgos esperables de la intervención y no atribuibles a una negligencia médica. Por el contrario, si la intervención no resulta en efectos adversos, solo debería otorgarse una indemnización al paciente si este logra demostrar al- guna afectación moral concreta y específica. No se trata de quitar relevancia a la infracción al derecho a consentir informadamente (en tanto la infracción puede tener alguna relevancia de otra índole, por ejemplo, administrativa), pero la responsabilidad civil solo opera cuando alguna consecuencia moral o material adversa puede ser identificada.

\section{Agradecimientos}

Este artículo está vinculado con el Proyecto Fondecyt de Iniciación 11180826, del que Gabriel Hernández es Investigador Responsable.

\section{Referencias}

1. Waldron J. How law protect dignity. The Cambridge Law Journal 2012; 71(1): 200-222.

2. McCrudden C. Human dignity in human rights interpretation. European Journal of International Law 2008; 19(4): 655-724.

3. Beauchamp T. Autonomy and consent. In: Miller F, Wertheimer A, eds. The ethics of consent. Nueva York: Oxford University Press; 2010: 55-78.

4. De la Maza I. Consentimiento informado, un poco de realismo. Revista de Derecho (Valdivia) 2017; 30(2): 111-131.

5. Le Tourneau P. Droit de la responsabilité et des contrats. Régimes d'indemnisation. 11 a ed. Paris: Dalloz; 2017.

6. Barros E. Tratado de responsabilidad extracontractual. 2a ed. Santiago: Editorial Jurídica del Chile; 2020.

7. Miller GA. The magical number seven, plus or minus two: some limits on our capacity for processing information. Psychological Review 1956; 63: 81-97.

8. Vidal Á. Responsabilidad civil médica. Santiago de Chile: Der Ediciones; 2018.

9. Pizarro C. La responsabilidad civil médica. Santiago de Chile: Thomson Reuters; 2017.

10. De la Maza I. Consentimiento informado, una visión panorámica. Ius et Praxis 2010; 16(2): 89-120.

11. Kleining J. The nature of consent. In: Miller F, Wertheimer A, eds. The ethics of consent. Nueva York: Oxford University Press; 2010: 3-24.

12. Perry S. Harm, history, and counterfactuals. San Diego Law Review 2003; 40: 1283-1314.

13. Oberdiek J. The moral significance of risking. Legal Theory 2012; 18: 339-356.

14. Hernández H. Consentimiento informado y responsabilidad penal médica: una relación ambigua y problemática. Cuadernos de Análisis Jurídico, Colección Derecho Privado 2010; VI: 167-185.

15. Pizarro C. En oposición al consentimiento hipotético informado. Revista de Derecho de la Pontificia Universidad Católica de Valparaíso 2015; 44: 97-120.

16. Reece H. Losses of chances in the law. The Modern Law Review 1996; 59(2): 188-206.

Recibido: 28 de septiembre de 2020

Aceptado: 30 de octubre de 2020 